\title{
Rare case of idiopathic sclerosing cholangitis, which was difficult to distinguish from cholangiocarcinoma: A case report
}

\author{
SHUNSUKE SHICHI $^{1}$, TAKAHIRO EINAMA ${ }^{1,2}$, MAYU SUZUKI $^{1}$, HIROKI MATSUI $^{1}$, RYO KANAZAWA $^{1}$, \\ KAZUAKI SHIBUYA ${ }^{1,2}$, TAKASHI SUZUKI ${ }^{1,2}$, FUMIHIKO MATSUZAWA ${ }^{1,2}$, KOHEI NAKACHI ${ }^{3}$, \\ TAKU HASHIMOTO ${ }^{1}$, NOBUO KONDO ${ }^{4}$, HIRONORI ABE ${ }^{1}$ and AKINOBU TAKETOMI ${ }^{2}$ \\ ${ }^{1}$ Department of Surgery, Hokkaido Social Work Association Obihiro Hospital, Obihiro, Hokkaido 080-0805; \\ ${ }^{2}$ Department of Gastroenterological Surgery I, Hokkaido University Graduate School of Medicine, Sapporo, \\ Hokkaido 060-8638; ${ }^{3}$ Department of Gastroenterology, Hokkaido Social Work Association Obihiro Hospital, \\ Obihiro, Hokkaido 080-0805; ${ }^{4}$ GeneticLab Co., Ltd., Sapporo, Hokkaido 060-0009, Japan
}

Received November 13, 2017; Accepted September 28, 2018

DOI: $10.3892 /$ etm.2018.6832

\begin{abstract}
It is often difficult to correctly diagnose patients who present with dilation of the bile duct. Cholangiocarcinoma, primary sclerosing cholangitis (PSC) and immunoglobulin (Ig)G4-related sclerosing cholangitis must be considered as potential diagnoses for these cases. The current study presents a 73-year-old female patient who presented with a high fever and abdominal pain. Contrast-enhanced computed tomography and magnetic resonance cholangiopancreatography revealed stenosis and dilation of the intrahepatic bile duct without solid components. It was suspected that the patient had intrahepatic cholangiocarcinoma. A left liver lobectomy, cholecystectomy and distal gastrectomy combined with a D2 lymph node dissection were performed. A pathological examination of the liver revealed increased fibrosis in the stroma, irregular bile duct dilation and clusters of inflamed lymph cells. No carcinoma or IgG4-positive plasma cells were observed and the typical findings of PSC were not detected. Based on these clinical and pathological results, the diagnosis was idiopathic sclerosing cholangitis, which is particularly rare. It is often difficult to preoperatively differentiate between cholangiocarcinoma and benign bile duct stenosis.
\end{abstract}

Correspondence to: Dr Shunsuke Shichi, Department of Surgery, Hokkaido Social Work Association Obihiro Hospital, Minami 9, Higashi 5, Obihiro, Hokkaido 080-0805, Japan

E-mail: shichishunsuke@gmail.com

Dr Takahiro Einama, Department of Gastroenterological Surgery I, Hokkaido University Graduate School of Medicine, Kita 14, Nishi 7, Kita-Ku, Sapporo, Hokkaido 060-8638, Japan

E-mail: titiuehahaue@hotmail.com

Key words: biliary duct stenosis, idiopathic sclerosing cholangitis, primary sclerosing cholangitis, immunoglobulin G4-related sclerosing cholangitis, cholangiocarcinoma

\section{Introduction}

It is difficult to rule out cholangiocarcinoma in patients with dilated bile ducts. Therefore, clinicians must consider primary sclerosing cholangitis (PSC) and immunoglobulin (Ig)G4-related sclerosing cholangitis (IgG4-SC) as potential diagnoses in such cases. The incidence of benign stricture of the hilar bile duct was reported to be approximately $10 \%$ of surgically resected cases involving a preoperative diagnosis of cholangiocarcinoma (1). Most benign hilar strictures detected in surgically resected cases involved PSC, IgG4-SC, or secondary sclerosing cholangitis (SSC). Here, we report a rare case of idiopathic sclerosing cholangitis, which was preoperatively diagnosed as cholangiocarcinoma. This case had some pathological features of SC, but it could not be classified into either of the reported types of benign SCs. So it was diagnosed as an idiopathic sclerosing cholangitis. We report this case including the pathological features about SCs.

\section{Case report}

A 73-year-old female presented with high fever and abdominal pain during follow-up after endoscopic mucosal resection (EMR) for gastric cancer. On admission, her laboratory findings were as follows: White blood cell count and $\mathrm{C}$ reactive protein level were elevated to $14,960 / \mu 1$ (neutrophils $=92.0 \%$ ) and $23.55 \mathrm{mg} / \mathrm{dl}$, respectively. In addition, her total bilirubin, aspartate aminotransferase, and alanine aminotransferase levels were elevated $0.62 \mathrm{mg} / \mathrm{dl}, 48$, and $39 \mathrm{U} / 1$, respectively. Carcinoembryonic antigen level was within the reference limits, whereas carbohydrate antigen level was slightly elevated, $68 \mathrm{U} / \mathrm{ml}$. Unfortunately, preoperative serum IgG4 was not measured. Postoperative serum IgG4 was measured periodically, but it was within normal range at 10 to $18 \mathrm{mg} / \mathrm{dl}$. Contrast-enhanced computed tomography and magnetic resonance cholangiopancreatography showed stenosis and dilation of the intrahepatic bile duct, in the left hepatic lobe; i.e., within segments 2 and 3 of the liver (Fig. 1). During percutaneous transhepatic biliary drainage, the contrast medium that was injected into the drainage tube 
could not pass into the center of the bile duct. Endoscopic retrograde cholangiopancreatography revealed obstruction of the intrahepatic bile duct. Scrape cytology of this narrow segment of the bile duct revealed some atypical cells, which were suggestive of hilar cholangiocarcinoma. In addition, follow-up upper endoscopy was conducted, and a biopsy of a scar on the patient's stomach caused by the previous EMR was carried out, which revealed tubular adenocarcinoma. According to these results, we suspected intrahepatic cholangiocarcinoma and diagnosed the patient with locally recurrent gastric cancer. Surgical resection of the liver and stomach were recommended. A lobectomy of the left side of the liver, cholecystectomy, and a distal gastrectomy combined with a D2 lymph node dissection were performed. The operation time was $10 \mathrm{~h}$ and $28 \mathrm{~min}$, and $840 \mathrm{ml}$ of intraoperative blood loss occurred. An examination of the surgical specimens showed that the bile duct was thickened and extended and the length of stenosis was $2 \mathrm{~cm}$, but there was no tumor. The patient did not suffer any complications during the postoperative period. A pathological examination of her liver showed marked fibrosis in the stroma and clusters of lymphoid cells. The bile duct exhibited irregular dilation and stenotic compression from the outside, but no thickening of the bile duct wall was noted. No carcinoma or IgG4-positive plasma cells were observed, and we did not detect the typical findings of PSC. The patient had no history of SSC (Fig. 2). Based on these clinical and pathological results, we made a diagnosis of idiopathic sclerosing cholangitis. Incidentally, the pathological findings from the patient's stomach showed early gastric carcinoma, but there was no evidence of a pathological relationship between the bile duct stenosis and the gastric cancer.

\section{Discussion}

It is difficult to differentiate between cholangiocarcinoma and benign bile duct stenosis preoperatively. In this case, we did not detect any obvious malignant findings; however, based on the progression of the intrahepatic bile duct stenosis and dilation, we suspected that the patient might have cholangiocarcinoma and decided to perform a lobectomy of the left side of the liver.

It remains difficult to distinguish bile duct malignancy from PSC or IgG4-SC based on imaging features alone. The presence of pancreatic abnormalities, including a peripancreatic rind, atrophication, abnormal enhancement, or high T2 signal intensity, strongly favors a diagnosis of IgG4-SC (2). However, benign bile duct stenosis is detected at a relatively high frequency during liver surgery (8-17\%) (1). Thus, additional diagnostic strategies are likely to be vital for distinguishing between these conditions (3).

PSC, IgG4-SC, and SSC are the most common causes of benign bile duct stenosis. PSC and IgG4-SC exhibit similar pathological features, but do differ in some aspects. For example, the pathological features of PSC include onion-skin fibrosis of the bile duct, whereas those of IgG4-SC include infiltration by IgG4-positive plasma cells, obliterative phlebitis, and storiform fibrosis (4). SSC displays suppurative inflammation and erosion of the bile duct mucosa during infections and ischemia. Some representative diseases, such as PSC, IgG4-SC, and SSC, cause benign bile duct stenosis and
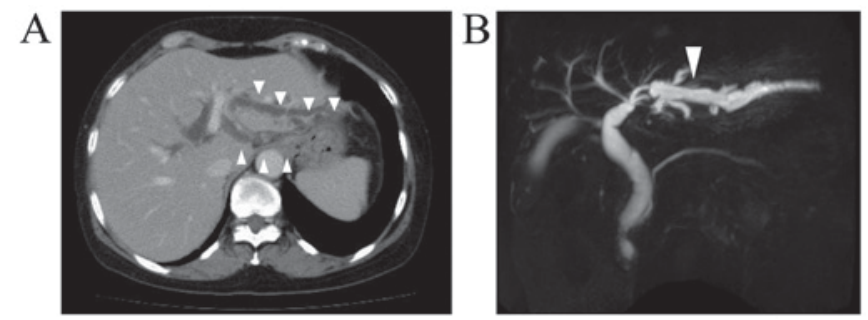

Figure 1. (A) Contrast-enhanced computed tomography and (B) magnetic resonance cholangiopancreatography of the abdomen revealed stenosis and dilation of the intrahepatic bile duct in the left hepatic lobe (segments 2 and 3 of the liver) as indicated by the arrowheads.
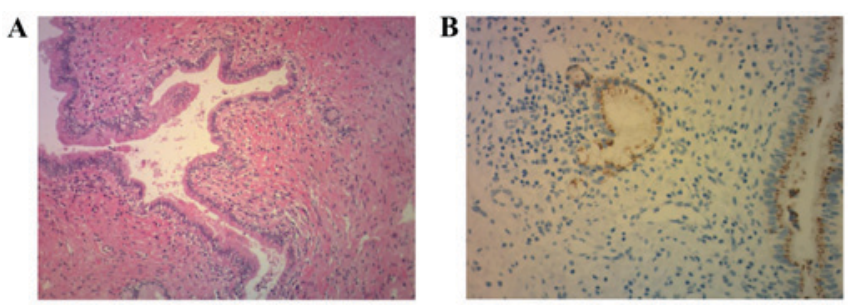

Figure 2. (A) Histopathological examination (hematoxylin and eosin staining) of the liver revealing marked fibrosis in the stroma, irregular bile duct dilation and clusters of lymph cells. The bile duct demonstrated irregular dilation or stenosis compressed from the outside but the walls of the bile duct were not thickened. No carcinoma was observed and the typical findings of PSC were not detected. Magnification x100. (B) IgG4 immunostaining of the specimen for IgG4 did not detect any IgG4-positive plasma cells. Magnification, x400. Ig, Immunoglobulin G; PSC, primary sclerosing cholangitis.

dilation. This case did not display the typical findings of PSC or IgG4-SC, and the patient did not have a history that was suggestive of SSC.

Fujita et al reported the pathological features of 5 cases of benign sclerosing cholangitis of unknown origin (1). Pathologically, fibroinflammatory changes and lymphoplasmacytic infiltration were observed in all 5 cases. Furthermore, these cases were classified into two types. One type exhibited a thickened bile duct wall and dense fibrosis as well as the formation of numerous lymphoid follicles with germinal centers under the mucosal layer. The other type involved a single focal stricture of the bile duct and subtle lymphoplasmacytic infiltration. The pathological features of our case included fibrosis and lymphoplasmacytic infiltration, which were seen in the previously reported cases, and lymphoid follicle formation, whereas thickening of the bile duct wall was absent. So, this case cannot be classified into either of the reported types of benign sclerosing cholangitis.

Therefore, it cannot be classified into typical benign bile duct stenosis, and as far as we know, no similar cases have been reported. So, we consider that this case was a rare case of idiopathic sclerosing cholangitis involving atypical pathological findings, such as benign bile duct stenosis.

In conclusion a rare case of idiopathic sclerosing cholangitis was determined. It is often difficult to differentiate between cholangiocarcinoma and benign bile duct stenosis preoperatively. Additional diagnostic strategies are likely to be vital for distinguishing between these diseases. 


\section{Acknowledgements}

Not applicable.

\section{Funding}

No funding was received.

\section{Availability of data and materials}

All data generated or analyzed during this study are included in this published article.

\section{Authors' contributions}

SS, TE, MS, HM, RK, KS, TS, FM, TH and HA performed the operation and perioperative medical treatment. KN performed preoperative examination and diagnosis. NK performed the histopathological examination of the liver. AT analyzed the pathological findings, assisted with patient diagnosis and was a major contributor in writing the manuscript. All authors read and approved the final manuscript.

\section{Ethics approval and consent to participate}

Written informed consent was obtained from the participating patient.

\section{Patient consent for publication}

Written informed consent for publication was obtained from the patient.

\section{Competing interests}

The authors declare that they have no competing interests.

\section{References}

1. Fujita T, Kojima M, Gotohda N, Takahashi S, Nakagohri T, Konishi M, Ochiai A and Kinoshita T: Incidence, clinical presentation and pathological features of benign sclerosing cholangitis of unknown origin masquerading as biliary carcinoma. J Hepatobiliary Pancreat Sci 17: 139-146, 2010.

2. Gardner CS, Bashir MR, Marin D, Nelson RC, Choudhury KR and Ho LM: Diagnostic performance of imaging criteria for distinguishing autoimmune cholangiopathy from primary sclerosing cholangitis and bile duct malignancy. Abdom Imaging 40: 3052-3061, 2015.

3. Kalaitzakis E, Levy M, Kamisawa T, Johnson GJ, Baron TH, Topazian MD, Takahashi N, Kanno A, Okazaki K, Egawa N, et al: Endoscopic retrograde cholangiography does not reliably distinguish IgG4-associated cholangitis from primary sclerosing cholangitis or cholangiocarcinoma. Clin Gastroenterol Hepatol 9: 800-803.e2, 2011.

4. Nakazawa T, Naitoh I, Hayashi K, Miyabe K, Simizu S and Joh T: Diagnosis of IgG4-related sclerosing cholangitis. World J Gastroenterol 19: 7661-7670, 2013.

(i) $\Theta$ This work is licensed under a Creative Common Attribution-NonCommercial-NoDerivatives 4.0 International (CC BY-NC-ND 4.0) License. 